\title{
Kicked particle dynamics in quantum graphs
}

\author{
J. Yusupov ${ }^{1}$, V. Eshniyozov ${ }^{2}$, O. Karpova ${ }^{1,2}$, D. Sh. Saidov ${ }^{3}$ \\ ${ }^{1}$ Turin Polytechnic University in Tashkent, 17. Niyazov Str., 100095, Tashkent, Uzbekistan \\ ${ }^{2}$ National University of Uzbekistan, 100174, Tashkent, Uzbekistan \\ ${ }^{3}$ Urganch Branch of Tashkent University of Information Technologies, Urganch, Uzbekistan \\ j.yusupov@polito.uz
}

PACS 03.65.Aa, 03.65.Ge

DOI 10.17586/2220-8054-2015-6-6-773-778

The quantum dynamics of a delta-kicked driven particle in a star-shaped network is studied by obtaining an exact solution for the time-dependent Schrödinger equation within a single kicking period. The timedependence of the average kinetic energy and the Gaussian wave packet evolution are analyzed.

Keywords: quantum graph,periodically kicked system,wave packet dynamics.

Received: 1 November 2015

\section{Introduction}

Particle dynamics in branched systems, such as networks and lattices are of importance in many topics of physics such as optics, acoustics, condensed matter and polymer physics. Particle and wave transport in such systems can be effectively modeled using the evolution equation on so-called metric graphs. Earlier, the Schrödinger equation on a metric graph was subject of extensive research (see review papers [1-8] and references therein). The nonlinear wave equation on metric graphs has also attracted much attention recently [9-16]. Graphs are the systems consisting of bonds which are connected at the vertices. The bonds are connected according to a rule, which is called the topology of a graph. The topology of a graph is given in terms of the adjacency matrix $[1,2]$ :

$$
C_{i j}=C_{j i}= \begin{cases}1, & \text { if } i \text { and } j \text { are connected; } i, j=1,2, \ldots, V . \\ 0, & \text { otherwise }\end{cases}
$$
$\left[0, L_{i j}\right]$.

A graph is called a metric graph when each of its bonds is associated with an interval

Earlier, quantum graphs were extensively studied in the context of quantum chaos theory [17-21]. Strict mathematical formulation of the boundary conditions was given by Kostrykin and Schrader [4]. Inverse problems on quantum graphs have been studied in Refs. [5]- [7]. An experimental realization of quantum graphs on (optical) microwave waveguide networks is discussed in the Ref. [8].

Despite the fact that different issues of quantum graphs and their applications have been discussed in the literature, the problem of graphs driven by external fields has not yet been treated.

In this paper, we address the problem of particle dynamics in periodically driven graphs by considering, as a perturbation, delta-kicking potential. The quantum dynamics of delta-kicked systems were extensively discussed in the context of quantum chaos and related issues [17] - [21]. A remarkable feature of the kicked quantum system is so-called quantum localization, which implies suppression of diffusive growth of the average kinetic 
energy as a function of time [18]. For classical kicked systems, energy grows linearly as a function of time $[17,18]$. Such a phenomenon can be considered as an analog of the Anderson localization [18]. We consider delta-kicked particle dynamics in the simplest graph topology, the so-called star graph. In particular, we study wave-packet evolution in such a system and the time-dependence of the average kinetic energy.

\section{Schrödinger equation on graphs}

Quantum particle dynamics on a graph is described by the one-dimensional Schrödinger equation $[1,2]$ (in the units $\hbar=2 m=1$ ):

$$
\frac{d^{2} \Psi_{b}(x)}{d x^{2}}=k^{2} \Psi_{b}(x), \quad b=(i, j),
$$

where $b$ denotes a bond connecting $i$ th and $j$ th vertices, and for each bond $b$, the component $\Psi_{b}$ of the total wave function $\Psi_{b}$ is a solution of the Eq.2. This equation is a multi-component equation where the components are related through boundary conditions, providing continuity and current conservation [1]:

$$
\left\{\begin{array}{l}
\bullet \text { Continuity, } \\
\left.\Psi_{i, j}\right|_{x=0}=\varphi_{i},\left.\quad \Psi_{i, j}\right|_{x=L_{i, j}}=\varphi_{j} \text { for all } i<j \text { and } C_{i, j} \neq 0 \\
\bullet \text { Current conservation, } \\
\left.\sum_{j<i} C_{i, j} \frac{d}{d x} \Psi_{j, i}(x)\right|_{x=L_{i, j}}+\left.\sum_{j>i} C_{i, j} \frac{d}{d x} \Psi_{i, j}(x)\right|_{x=0}=-\lambda_{i} \varphi_{i} .
\end{array}\right.
$$

Here, the parameters $\lambda_{i}$ are free parameters which determine the type of boundary conditions. In particular, the special case of zero $\lambda_{i}$ 's corresponds to the Neumann boundary conditions. The Dirichlet boundary conditions correspond to the case when all the $\lambda_{i}=\infty$.

The eigenfunctions obeying continuity conditions can be written as:

$$
\Psi_{i, j}=\frac{C_{i, j}}{\sin k L_{i, j}}\left(\varphi_{i} \sin k\left(L_{i, j}-x\right)+\varphi_{j} \sin k x\right)
$$

while current conservation gives:

$$
\sum_{j \neq i} \frac{k C_{i, j}}{\sin k L_{i, j}}\left(-\varphi_{i} \cos k L_{i, j}+\varphi_{j}\right)=\lambda_{i} \varphi_{i} .
$$

Corresponding eigenfunctions can be found from the following quantization condition:

$$
\operatorname{det}\left(h_{i, j}(k)\right)=0
$$

where

$$
h_{i, j}(k)= \begin{cases}-\sum_{m \neq i} C_{i, m} \cot k L_{i, m}-\frac{\lambda_{i}}{k}, & i=j \\ C_{i, j} \sin ^{-1} k L_{i, j}, & i \neq j\end{cases}
$$

For the star graph, the boundary conditions can be written as [22]:

$$
\left\{\begin{array}{l}
\left.\Psi_{1}\right|_{y=0}=\left.\Psi_{2}\right|_{y=0}=\ldots=\left.\Psi_{N}\right|_{y=0}, \\
\left.\Psi_{1}\right|_{y=L_{1}}=\left.\Psi_{2}\right|_{y=L_{2}}=\ldots=\left.\Psi_{N}\right|_{y=L_{N}}=0, \\
\left.\sum_{j=1}^{N} \frac{d}{d y} \Psi_{j}\right|_{y=0}=0
\end{array}\right.
$$

where $N$ is the number of bonds emanating from the central vertex. 
In the case of the star graph, the energy spectrum can be found from the following spectral equation [22]:

$$
\sum_{j=1}^{N} \tan ^{-1}\left(k_{n} L_{j}\right)=0
$$

while the corresponding eigenfunctions are written as [22]:

$$
\Psi_{j}^{(n)}=\frac{B_{n}}{\sin \left(k_{n} L_{j}\right)} \sin \left(k_{n}\left(L_{j}-y\right)\right)
$$

where the normalization coefficients are given by:

$$
B_{n}=\left[\sum_{j}\left(L_{j}+\sin \left(2 k_{n} L_{j}\right)\right) \sin ^{-2}\left(k_{n} L_{j}\right) / 2\right]^{-1 / 2}
$$

\section{Kicked star graph}

Consider a quantum particle on a primary star graph, i.e. on a graph with three bonds, in the presence of an external time-periodic potential. Such system is described by the following time-dependent Schrödinger equation:

$$
i \frac{\partial \Psi_{b}(x, t)}{\partial t}=\left[-\frac{\partial^{2}}{\partial x^{2}}-\varepsilon \cos x \delta_{T}(t)\right] \Psi_{b}(x, t), \quad b=1,2,3 .
$$

where:

$$
\delta_{T}(t)=\sum_{l=-\infty}^{\infty} \delta(t-l T)
$$

with $T$ being the kicking period.

Eq.12 can be analytically integrated over a single kicking period. To do this, we note that the solution of Eq. 12 can be expanded in terms of complete set $\left\{\phi_{b}^{(n)}\right\}(b=1,2,3)$, of solutions of Eq. 2 as:

$$
\Psi_{b}(x, t)=\sum_{n} A_{n}(t) \phi_{b}^{(n)}(x)
$$

Integrating Eq.12 over a single period $T$, using the same prescription as in the case of the kicked rotor $[17,18]$ for the time evolution of $A_{n}(t)$, during one kicking, period we have:

$$
A_{m}(t+T)=\sum_{n} A_{n}(t) V_{m n} e^{-i E_{n} T}
$$

where $E_{n}$ represents the eigenvalues of unperturbed star graph:

$$
V_{m n}=\sum_{b=1}^{3} \int_{0}^{L_{b}} \phi_{b}^{(m)^{*}}(x) e^{i \varepsilon \cos x} \phi_{b}^{(n)}(x) d x .
$$

Using Eq.15, we can compute wave function for an arbitrary number of kicks and average kinetic energy as:

$$
\langle E(t)\rangle=-\frac{1}{2} \sum_{b=1}^{3} \int_{0}^{L_{b}} \Psi_{b}^{*}(x, t) \frac{\partial^{2} \Psi_{b}(x, t)}{\partial x^{2}} d x .
$$

In Fig. 1, $\langle E(t)\rangle$ is plotted as a function of time for different kicking strength values.

As is seen from these plots, the average energy is a periodic function of time with a period much longer than that of the kicking period. This behavior is completely different than 


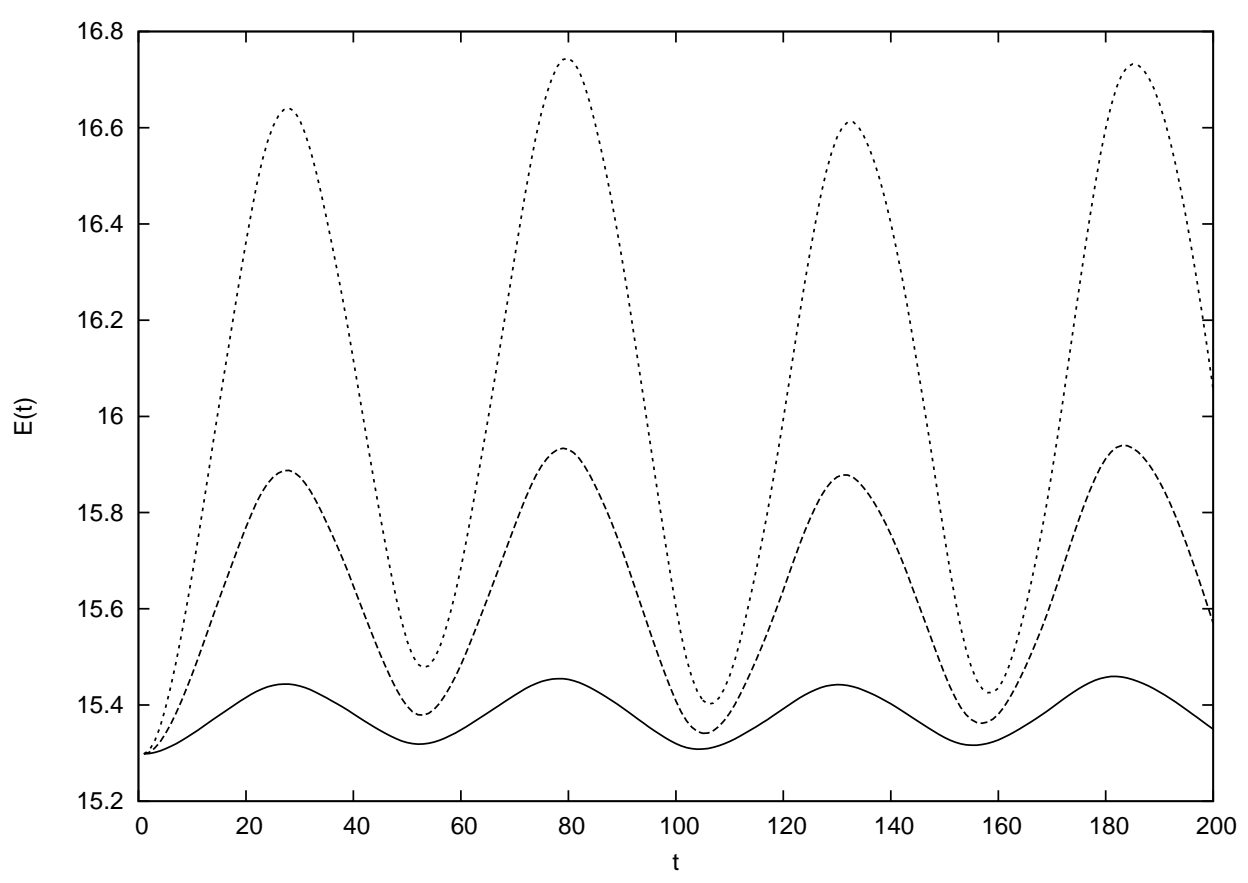

FIG. 1. Average kinetic energy as a function of time for different kicking strength values: $\varepsilon=0.3$ (dotted), $\varepsilon=0.2$ (dashed) and $\varepsilon=0.1$ (solid) at the kicking period, $T=0.001$

that of the kicked rotor case [18] and the kicked one dimensional box [20]. Such a periodicity may be caused by a more complicated structure for the graph, which implies different (than those for kicked rotor or box) boundary conditions in the Schrödinger equation.

Furthermore, we consider wave packet evolution in kicked star graph by taking the wave function at $t=0$ (for the first bond) as the following Gaussian wave packet:

$$
\Psi_{1}(x, 0)=\Phi(x)=(\sqrt{2 \pi} \sigma)^{-1 / 2} \mathrm{e}^{-(x-m u)^{2} / 4 \sigma},
$$

with $\mu$ and $\sigma$ being the initial position and the width of the packet. For other bonds, initial wave function is assumed to be zero, i.e. $\Psi_{2}(x, 0)=\Psi_{3}(x, 0)=0$. Then for the initial values of the wave functions $\Psi_{b}(x, t)$, the expansion coefficients at $t=0$ can be written as:

$$
A_{n}(0)=\int_{0}^{L_{1}} \Phi(x) \phi_{1}^{(n)^{*}}(x) d x .
$$

Fig. 2 presents the time evolution of the Gaussian wave packet on kicked star graph for kicking parameters $\varepsilon=0.1$ and $T=0.01$ at time moments $t=100 T, 300 T$ and $500 T$. As these plots show, complete dispersion of the wave packet is not possible, even for a high number of kicks, due to the confined nature of the system. Also, wave packet revival can be observed in such systems. 
(a)

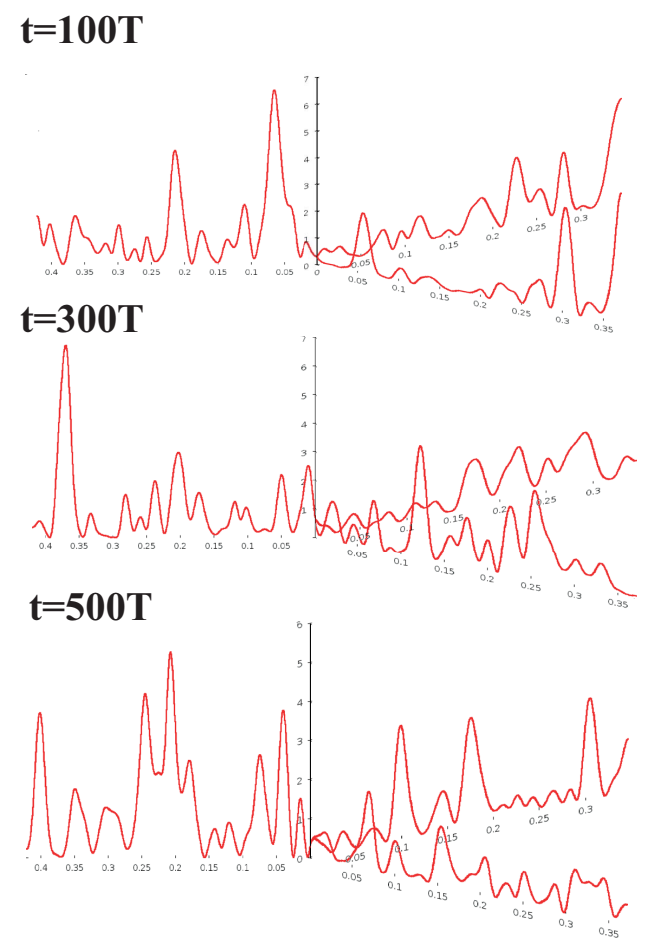

(b)

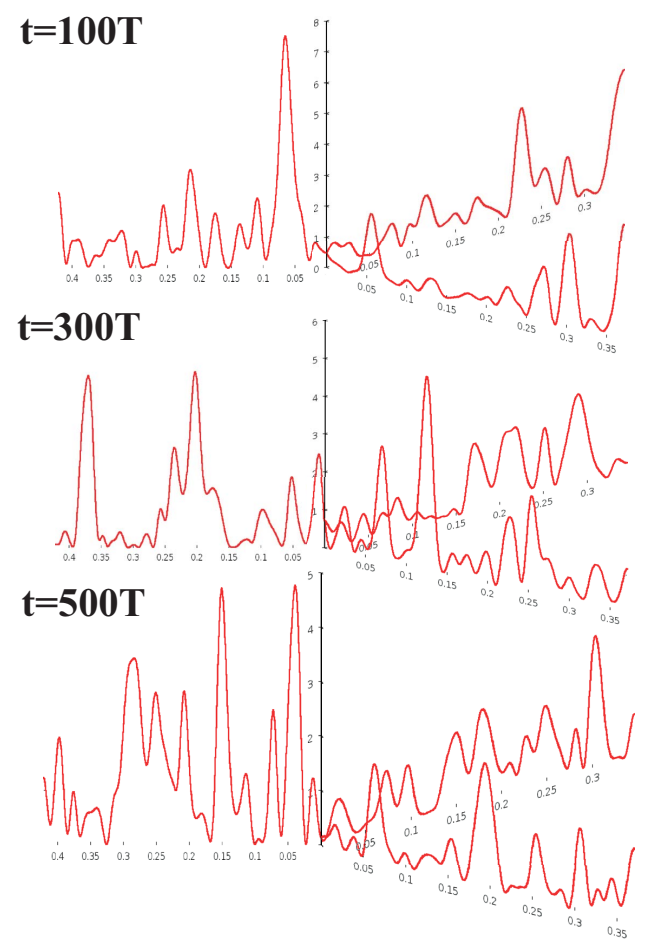

Fig. 2. Time evolution of the Gaussian wave packet in kicked quantum star graph for kicking parameters $\varepsilon=0.1, T=0.001$

\section{Conclusion}

In this work, we have studied the quantum dynamics of a delta kicked particle in a star graph driven by external periodic delta-kicking field by considering the time-dependence of the average kinetic energy and wave packet evolution. It was shown that the average kinetic energy of a kicked particle in a star graph is a periodic function of time. The amplitude and period of the average kinetic energy depend on the kicking parameters (kicking strength and period). By tuning the kicking parameters, it is possible to find a regime when the average kinetic energy grows monotonically over time. The absence of complete wave packet dispersion was also shown for this system. The results can be used for the realization of quantum Fermi acceleration in nanoscale networks and achieving tunable electronic transport in nanoscale devices.

\section{References}

[1] T. Kottos, U. Smilansky. Periodic Orbit Theory and Spectral Statistics for Quantum Graphs. Ann.Phys., 1999, 274(1), P. 76-124.

[2] S. Gnutzmann, U. Smilansky. Quantum graphs: Applications to quantum chaos and universal spectral statistics. Adv.Phys., 2006, 55(5-6) P. 527-625.

[3] S. Gnutzmann, J.P. Keating, F. Piotet. Eigenfunction statistics on quantum graphs. Ann.Phys., 2010, 325(12) P. 2595-2640.

[4] R. Kostrykin, R. Schrader. Kirchhoff's rule for quantum wires. J. Phys. A: Math. Gen., 1999, 32, P. 595.

[5] B. Gutkin, U. Smilansky. Can one hear the shape of a graph? J. Phys A., 2001, 31, P. 6061.

[6] J. Boman, P. Kurasov. Symmetries of quantum graphs and the inverse scattering problem. Adv. Appl. Math., 2005, 35(1), P. 58-70. 
[7] T. Cheon, P. Exner, O. Turek. Approximation of a general singular vertex coupling in quantum graphs. Ann.Phys., 2010, 325 P. 548-578.

[8] O. Hul, S. Bauch, P. Pakonski, N. Savytskyy, K. Zyczkowski, L. Sirko. Experimental simulation of quantum graphs by microwave networks. Phys. Rev. E, 2004, 69, P. 056205.

[9] Z. Sobirov, D. Matrasulov, K. Sabirov, S. Sawada, K. Nakamura. Integrable nonlinear Schrodinger equation on simple networks: Connection formula at vertices. Phys.Rev.E, 2010, 81, P. 066602.

[10] R. Adami, C. Cacciapuoti, D. Finco, D. Noja. Fast solitons on star graphs. Rev. Math. Phys., 2011, 23(04), P. 409-451.

[11] R. Adami, C. Cacciapuoti, D. Finco, D. Noja. Stationary states of NLS on star graphs. Europhys. Lett., 2012, 100, P. 10003

[12] R. Adami, C. Cacciapuoti, D. Finco, D. Noja. On the structure of critical energy levels for the cubic focusing NLS on star graphs. J. Phys. A: Math. Theor., 2012, 45, P. 192001.

[13] R. Adami, D. Noja, and C. Ortoleva. Orbital and asymptotic stability for standing waves of a nonlinear Schrödinger equation with concentrated nonlinearity in dimension three. J. Math. Phys., 2013, 54, P. 013501.

[14] K. Sabirov, Z. Sobirov, D. Babajanov, D. Matrasulov. Stationary Nonlinear Schrödinger Equation on Simplest Graphs. Phys.Lett. A, 2013, 377, P. 860.

[15] D. Noja. Nonlinear Schrödinger equation on graphs: recent results and open problems. Philos. Trans. R. Soc. A, 2013, 372, P. 20130002.

[16] C. Cacciapuoti, D. Finco, D. Noja. Topology-induced bifurcations for the nonlinear Schrdinger equation on the tadpole graph. Phys. Rev. E, 2015, 91, P. 013206.

[17] G. Casati, B.V. Chirikov, J. Ford, F.M. Izrailev. Lecture Notes in Physics, Vol. 93, P. 334 (Springer, Berlin, 1979).

[18] G.M. Izrailev. Simple models of quantum chaos: spectrum and eigenfunctions. Phys. Rep., 1990, 196, P. 299-392.

[19] A. Buchleitner, D. Delande, J. Zakrzewski. Non-dispersive wave packets in periodically driven quantum systems. Phys. Rep., 2002, 368(5), P. 409-547.

[20] R. Sankaranarayanan, V. Sheorey. Accelerator modes of square well system. Phys. Lett. A, 2005, 338, P. 288-296.

[21] F. Haug, M. Bienert, W.P. Schleich, T.H. Seligman, M.G. Raizen. Motional stability of the quantum kicked rotor: A fidelity approach. Phys. Rev. A, 2005, 71, P. 043803.

[22] J.P. Keating. Fluctuation statistics for quantum star graphs. Quantum graphs and their applications. Contemp. Math., 2006, 415, P. 191-200. 